\title{
The complex mass from Henstock-Kurzweil-Feynman-Pardy integral
}

\author{
Miroslav Pardy \\ Department of Physical Electronics \\ Masaryk University \\ Kotlářská 2, 61137 Brno, Czech Republic \\ e-mail:pamir@physics.muni.cz
}

\begin{abstract}
The Feynman integral is generalised so as to involve the random fluctuations of vacuum, from this integral the generalized Schrödinger equation is derived and the energy spectrum for the Coulomb potential determined.
\end{abstract}

\section{Introduction}

The purpose of this paper is to generalize the Feynman integral over paths in the case, where we consider random fluctuations of vacuum, and to derive the generalized Schrödinger equation. The article is the improved version of the author article (Pardy, 1973), where the Henstock-Kurzweil integral (Henstock, 1946; Morales, et al., 2017) is not involved in it. 
First, let us remember Feynman's fictitious experiment by means of which he arrives at the integral over paths (Feynman \& Hibbs, 1965). A source of electrons is considered in this experiment and a movable detector is fixed at a certain distance from the source. Between the source and the detector is put a screen with two holes, 1 and 2, in it. Now, we investigate the probability that an electron will arrive in the detector at various vertical distances $x$ from the source. If we block hole 2 , the particle will pass through hole 1 , and if we block hole 1 the particle will go through hole 2 . The probability $P_{1}(x)$ to find a particle at point $\mathrm{x}$ will be given in the first case like this:

$$
P_{1}(x)=\left|\phi_{1}(x)\right|^{2},
$$

where $\phi_{1}(x)$ is the complex function called the probability amplitude corresponding to the path going through hole 1 in the detector. In the second case the probability to find a particle at point $x$ is $P_{2}(x)$ and it holds good:

$$
P_{2}(x)=\left|\phi_{2}(x)\right|^{2},
$$

where $\phi_{2}(x)$ is the probability amplitude corresponding to the path going through hole 2 in the detector.

If we leave open both holes, the probability of finding a particle at point $x$ will be given like this:

$$
P(x)=\left|\phi_{1}(x)+\phi_{2}(x)\right|^{2} .
$$

Now, let us consider that there is not only one screen between the source and the detector but that there are $\mathrm{N}$ screens, each of them having $M$ holes, with width $\Delta\left(A_{i}, B_{i}\right)_{j}$, where $A_{i}, B_{i}$ are $x$-coordinates of the borders of the holes, $j$ being an index belonging to the $j$ th screen, $j=1,2, \cdots, N$ and $i$ being an index belonging to the $i$ th hole, $i=1,2, \cdots, M ; \Delta$ denotes the length of the interval $($,$) . We can say that the trajectory of the$ particle rises in such a way that the particle goes through interval $\left(a_{1}, b_{1}\right)$ at the time $t_{1}$, through the interval $\left(a_{2}, b_{2}\right)$ at the time $t_{2} \cdots$, through the interval $\left(a_{n}, b_{n}\right)$ at the time $t_{n}$, where $\left(a_{i}, b_{i}\right)$ are intervals from the set of intervals $\left(A_{i}, B_{i}\right)_{j}$. According to Feynman, the probability amplitude $\phi(x)$ belongs to this trajectory. If we choose another set of numbers $t_{k},\left(a_{k}, b_{k}\right)$, we get other alternative path of the particle.

It is obvious that there are a great many of these alternative trajectories, and therefore the total amplitude for the process is the sum of the amplitudes for each route considered separately. For the case $N \rightarrow \infty$, 
$M \rightarrow \infty$, all trajectories will be continuous functions of the time $t$, and the total probability amplitude for the transition of a particle from point $a$ to point $b$ is as follows:

$$
U(b, a)=\sum_{a \rightarrow b} \phi[x(t)]
$$

where $a \rightarrow b$ in $\sum$ means summation over all trajectories from $a$ to $b$ and $x(t)$ is the $x$-coordinate of a path. The amplitude is postulated as follows:

$$
\phi[x(t)]=\frac{1}{A} \exp \{(i / \hbar) S[x(t)]\},
$$

where

$$
S=\int_{t_{a}}^{t_{b}} L[\dot{x}(t) ; x(t)] d t
$$

is the classical Hamilton-Jacobi action function and $L$ is the Lagrange function.

Feynman \& Hibbs (1965) have shown that the probability amplitude $U\left(x, x_{0}\right)$ concerning the transition of the particle from point $x_{0}$ to point $x$ satisfies the integral equation

$$
U\left(x, x_{0}\right)=\int U\left(x, x_{1}\right) U\left(x_{1}, x_{0}\right) d x_{1} .
$$

If we put $U\left(x, x_{0}\right)=\psi(x), U\left(x_{1}, x_{0}\right)=\psi\left(x_{1}\right)$, we get

$$
\psi(x)=\int U\left(x, x_{1}\right) \psi\left(x_{1}\right) d x_{1},
$$

or, generally

$$
\psi(x, t)=\int U\left(x, t ; x_{1}, t_{1}\right) \psi\left(x_{1}, t_{1}\right) d x_{1}
$$

and the function $\psi(x, t)$ may be interpreted as the wave function of quantum mechanics.

For an infinitesimal transition from point $x_{k}$ and time $k_{k}$ in point $x_{k+1}$ and time $t_{k+1}$, we can write (Blokhintsev, 1966):

$$
U\left(x_{k+1}, k_{k+1} ; x_{k}, t_{k}\right)=\frac{1}{A} \exp \left\{(i / \hbar) S\left[x_{k+1}, x_{k}, \Delta t\right]\right\},
$$

where

$$
S\left[x_{k+1}, x_{k}, \Delta t\right]=\left[\frac{m}{2}\left(\frac{x_{k+1}-x_{k}}{\Delta t}\right)-V\left(x_{k+1}\right) \Delta t\right] \Delta t,
$$

where $\Delta t=t_{k+1}-t_{k}, t_{k+1}>t_{k}$. 


\section{Random fluctuations of the Vacuum in the integral over paths}

Let us notice term eq. (4)

$$
U\left(x_{1}, x_{0}\right)=\sum_{x_{0} \rightarrow x} \phi[x(t)] .
$$

We can see that all amplitudes $\exp (i / \hbar) S[x(t)]$ are multiplied only by a constant $1 / A$. In other words, the Feynman sum is constructed in such a way that all trajectories are considered equivalent and therefore equally probable.

The question arises, as to how the formulation of the problem must be changed if we include the random fluctuations of the vacuum. It is obvious that these fluctuations cause perturbations of the considered trajectories and, no doubt, in such a way that some trajectories will be less probable and others more probable. We arrive at this conclusion because it is obvious from the viewpoint of statistical mechanics that the particle will perform the Brownian motion as a result of accidental collisions.

This motion is described by the Wiener measure $\mu$ (Gelfand \& Yaglom, 1956):

$$
\begin{gathered}
\mu=\int_{a_{1}}^{b_{1}} \int_{a_{2}}^{b_{2}} \cdots \int_{a_{n}}^{b_{n}} \times \\
\frac{\exp \left\{-\frac{\left(x_{1}-x_{0}\right)}{4 D\left(t_{1}-t_{0}\right)}-\frac{\left(x_{2}-x_{1}\right)}{4 D\left(t_{2}-t_{1}\right)}-\cdots-\frac{\left(x_{n}-x_{n-1}\right)}{4 D\left(t_{n}-t_{n-1}\right)}\right\}}{\left[(4 \pi D)^{n}\left(t_{1}-t_{0}\right)\left(t_{2}-t_{1}\right) \cdots\left(t_{n}-t_{n-1}\right)\right]^{1 / 2}} d x_{1} d x_{2} \cdots d x_{n},
\end{gathered}
$$

the physical meaning of measure $\mu$ being as follows: measure $\mu$ determines the probability that the particle goes through the interval $\left(a_{1}, b_{1}\right)$ at the time $t_{1}$, through the interval $\left(a_{2}, b_{2}\right)$ at the time $t_{2}, \cdots$, through the interval $\left(a_{n}, b_{n}\right)$ at the time $t_{n}, t_{1}<t_{2}<t_{3}<\cdots<t_{n}$. The constant $D$ in the formula (12) is the so-called coefficient of diffusion, and it involves size, mass, etc. of the elementary particle.

In consideration of what was said, we are forced to make an "intuitive" assumption that the amplitude $U\left(x, x_{0}\right)$ will involve the effect of fluctuations after multiplying term $[\exp (i / \hbar) S] / A$ by $\mu$, where $\mu$ is given by eq. (12). For the infinitesimal transition from point $x_{k}$ to point $x_{k+1}$ we can write (Montroll, 1952):

$$
\mu\left(x_{k+1}, x_{k}\right)=\frac{1}{(4 \pi D \Delta t)^{1 / 2}} \exp \left[-\left(x_{k+1}-x_{k}\right)^{2} / 4 D \Delta t\right]
$$


and therefore for $U\left(x_{k+1}, t_{k+1 ; x_{k}, t_{k}}\right)$ we get

$$
\begin{gathered}
U\left(x_{k+1}, t_{k+1 ; x_{k}, t_{k}}\right) \rightarrow \\
\frac{1}{A(4 \pi D \Delta t)^{1 / 2}} \exp \left[-\frac{\left(x_{k+1}-x_{k}\right)^{2}}{4 D \Delta t}\right] \exp \left\{(i / \hbar) S\left[x_{k+1}, x_{k}, \Delta t\right]\right\} .
\end{gathered}
$$

Now we can arrive at deriving the generalised Schrödinger equation.

\section{The Generalised Schrödinger Equation}

We have said in the introduction that $\psi$ satisfies the equation

$$
\psi\left(x_{k+1}, t_{k+1}\right)=\int U\left(x_{k+1}, t_{k+1} ; x_{k}, t_{k}\right) \psi\left(x_{k}, t_{k}\right) d x_{k} .
$$

If we insert eq. (14) into eq. (15) and put

$$
\begin{gathered}
x_{k+1}-x_{k}=\zeta ; \quad x_{k+1}=x ; \quad t_{k}=t ; \quad t_{k+1}-t_{k}=\delta, \\
\alpha=\frac{m}{2 \hbar \delta} ; \quad \beta=\frac{1}{4 D \delta} \quad \exp [-(i / \hbar) V(x) \delta] \approx 1-\frac{i}{\hbar} V(x) \delta
\end{gathered}
$$

we get from eq. (15) the following equation

$$
\begin{gathered}
\psi(x, t+\delta)=\frac{1}{A}\left(\frac{\beta}{\pi}\right)^{1 / 2} \\
\int_{-\infty}^{\infty} \exp \left(i \alpha \zeta^{2}-\beta \zeta^{2}\right)\left[1-\frac{i \delta}{\hbar} V(x)\right] \psi(x-\zeta, t) d \zeta .
\end{gathered}
$$

Obviously

$$
\begin{gathered}
\psi(x, t+\delta)=\psi(x, t)+\delta \frac{\partial \psi}{\partial t}+\cdots \\
\psi(x-\zeta, t)=\psi(x, t)-\zeta \frac{\partial \psi}{\partial x}+\zeta^{2} \frac{1}{2} \frac{\partial^{2} \psi}{\partial x^{2}} \cdots
\end{gathered}
$$

We can write instead of (18) that

$$
\psi(x, t)+\delta \frac{\partial \psi}{\partial t}+\cdots=\frac{1}{A}\left(\frac{\beta}{\pi}\right)^{1 / 2} \int_{-\infty}^{\infty} \exp \left(i \alpha \zeta^{2}-\beta \zeta^{2}\right)\left[\psi(x, t)-\zeta \frac{\partial \psi}{\partial x}+\right.
$$




$$
\left.\frac{1}{2} \frac{\partial^{2} \psi}{\partial x^{2}} \zeta^{2}-\frac{i \delta}{\hbar} V(x) \psi+\frac{i \delta}{\hbar} V(x) \zeta \frac{\partial \psi}{\partial x}-\frac{i \delta}{\hbar} V(x) \frac{1}{2} \frac{\partial^{2} \psi}{\partial^{2} x} \zeta^{2}\right] d \zeta
$$

After modification of equation (20) we get

$$
\begin{gathered}
\psi(x, t)+\delta \frac{\partial \psi}{\partial t}+\cdots=\left(\frac{\beta}{\pi}\right)^{1 / 2} \times \\
\times\left\{\frac{J_{1}}{A} \psi-\frac{J_{2}}{A} \frac{\partial \psi}{\partial x}+\frac{J_{3}}{2 A} \frac{\partial^{2} \psi}{\partial x^{2}}+\frac{i \delta J_{1}}{A \hbar} V \psi+\frac{i \delta J_{2}}{A \hbar} V \frac{\partial \psi}{\partial x}-\frac{i \delta J_{3}}{2 A \hbar} V \frac{\partial^{2} \psi}{\partial x^{2}}\right\} .
\end{gathered}
$$

where

$$
\begin{gathered}
J_{1}=\int_{-\infty}^{\infty} \exp \left(i \alpha \zeta^{2}-\beta \zeta^{2}\right) d \zeta \\
J_{2}=\int_{-\infty}^{\infty} \exp \left(i \alpha \zeta^{2}-\beta \zeta^{2}\right) \zeta d \zeta \\
J_{3}=\int_{-\infty}^{\infty} \exp \left(i \alpha \zeta^{2}-\beta \zeta^{2}\right) \zeta^{2} d \zeta
\end{gathered}
$$

We can see that there is an odd function in $J_{2}$. Therefore, $J_{2}=0$. For two remaining integrals we get, according to Gradshtein \& Ryzhik (1963):

$$
\begin{gathered}
J_{1}=\frac{\sqrt{\pi}}{\left(\alpha^{2}+\beta^{2}\right)^{1 / 4}} \exp [(i / 2) \arctan (\alpha / \beta)], \\
J_{3}=\frac{\sqrt{\pi}}{2\left(\alpha^{2}+\beta^{2}\right)^{3 / 4}} \exp [(3 i / 2) \arctan (\alpha / \beta)] .
\end{gathered}
$$

We easily find that

$$
\begin{gathered}
\frac{J_{3}}{J_{1}}=-\frac{2 i \delta}{\hbar} \gamma, \\
\gamma=\frac{\hbar D}{\left[1+(\alpha / \beta)^{2}\right]}\left[i-\frac{\alpha}{\beta}\right],
\end{gathered}
$$

and $\gamma$ is not dependent on $\delta$.

If we put

$$
A=J_{1}\left(\frac{\beta}{\pi}\right)^{1 / 2}
$$

we can write instead of (21) that 


$$
\psi(x, t)+\delta \frac{\partial \psi}{\partial t}+\cdots=\psi-\left\{\frac{i \delta}{\hbar} V \psi-\frac{i \delta}{\hbar} \gamma \frac{\partial^{2}}{\partial x^{2}}-\frac{i \delta^{2}}{\hbar^{2}} \delta^{2} \gamma \frac{\partial^{2}}{\partial x^{2}}\right\} .
$$

After comparing the coefficients with $\delta$ and modification, we get

$$
i \hbar \frac{\partial \psi}{\partial t}=\gamma \frac{\partial^{2} \psi}{\partial x^{2}}+V \psi
$$

The last equation is the generalized Schrödinger equation for the onedimensional case only. For the case of three dimensions, we can write

$$
i \hbar \frac{\partial \psi}{\partial t}=\gamma \nabla^{2} \psi+V \psi .
$$

We can easily verify that

$$
\lim _{D \rightarrow \infty} \gamma=-\frac{\hbar^{2}}{2 m} .
$$

Therefore, equation (32) converges to the Schrödinger equation for $D \rightarrow \infty$.

\section{The Energy Spectrum for the Coulomb Potential}

Let us look for the solution of equation (32) in the form

$$
\psi=\exp [-(i / \hbar) \varepsilon t) \varphi
$$

After inserting eq. (34) into eq. (31) we get

$$
\varepsilon \varphi=\gamma \nabla^{2} \varphi+V \varphi
$$

By putting

$$
\gamma=-\frac{\hbar^{2}}{2 m^{\prime}}
$$

and

$$
m^{\prime}=m+\delta m,
$$

where

$$
\delta m=\frac{i \hbar}{2 D} .
$$


we can write instead of equation (38)

$$
\varepsilon \varphi=\frac{\hbar^{2}}{2 m^{\prime}} \nabla^{2} \varphi+V \varphi,
$$

where $m^{\prime}$ is the complex quantity.

The fact that the mass $m^{\prime}$ is the complex quantity we interpret in such a way that we consider the complex mass as the mathematical notion suitable for the physical application. We arrive at this interpretation after comparing it with other authors who use in a similar way the complex quantities which have no immediate physical content. Popov et al. (1967) have introduced imaginary time for the description of the quasistationary processes. The complex potentials for describing the atomic collisions has been used by Mizuno \& Chen (1971). Lee \& Wick (1969) have arrived at the complex mass in the consequence of the indefinite metric, and Yamamoto \& Kudo (1971) have considered the complex mass to be an integral part of quantum theory of fields. So, if the complex mass is of physical meaning it means that path integral involving random fluctuation of vacuum is also of physival meaning.

Now, we can start with the determination of the energy spectrum.

We know from quantum mechanics (Merzbacher, 1970) that the energy spectrum for the radial equation for equation (35) with the Coulomb potential

$$
V(r)=-\frac{Z e^{2}}{r}
$$

is given by the following expression:

$$
\varepsilon_{n}=-\frac{Z^{2} m^{\prime} e^{4}}{2 n^{2} \hbar^{2}} .
$$

By putting

$$
E_{n}=-\frac{Z^{2} m e^{4}}{2 n^{2} \hbar^{2}},
$$

where $m$ is the classical mass of the electron, we get with regard to eq. (37) and eq. (38):

$$
\varepsilon_{n}=E_{n}\left(1+\frac{i \hbar}{2 D}\right) .
$$

In the end we have for the wave function the expression

$$
\psi=\exp \left[-(i / \hbar) E_{n} t\right] \cdot \exp \left[-\left(\Gamma_{n} / 2\right) t\right] \varphi
$$


where

$$
\Gamma_{n}=-\frac{E_{n}}{m D},
$$

that is

$$
\Gamma_{n}=\frac{e^{4}}{2 n^{2} \hbar^{2} D} .
$$

We note that the motion of the electron in the Coulomb potential is not stationary but quasistationary as a result of the term $\exp \left[-\left(\Gamma_{n} / 2\right) t\right]$ in the wave function.

It is obvious that the random vacuum fluctuations will also effectively modify the potential $V(r)$ in which the electron moves. The classical derivation of this effect with the electromagnetic fluctuations was performed by Welton (1948) and energy shift of H-atom electrons due to Gibbons-Hawking thermal bath was calculated by author (Pardy, 2016)

\section{References}

Blokhintsev, D. i. (1966). The Fundamental Questions of Quantum Mechanics. Nauka Moscow, Chapter 16.2. (in Russian).

Feynman, R. P. and Hibbs, A. R. (1965). Quantum Mechanics and Path Integrals.(McGraw- Hill, New York).

Gelfand, I. M. and Yaglom, A. M. (1956). Integration in the functional spaces and its application in quantum physics, Uspekhi matematicheskikh nauk., 11, No. 1(67), 77-114.

Gradshtein, I. S. and Ryzhik, I. M. (1963). Tables of lntegrals, Sums, Series and Products, (GIFML, Moscow) pp. 498 and 514.

Henstock, R. (1946). On Interval Functions and their Integrals. Journal of the London Mathematical Society, 21, No. 3, 204-209.

Lee, T. D. and Wick, G. C. (1969). Negative metric and the unitarity of the S-matrix, Nuclear Physics, B9, 209.

Merzbacher, E. (1970).Quantum Mechanics (John Wiley \& Sons), New York, p. 204.

Mizuno, J. and Chen, J. C. Y. (1971). Theoretical Investigation of Complex Potentials for Atomic Collisions. II. Semiclassical Descriptions of Atomic Collisions Physical Review, A4, 1500. 
Montroll, E. W. (1952). Markoff chains, Wiener integrals, and quantum theory, Communications on Pure and Applied Physics, 5, 415.

Morales, M. G. and Gaitn, R. (2017). Feynman's path integral seen as a Henstock integral, Journal of Physics, Conf. Series 912, doi: 10.1088/17426596/912/1/012014.

Pardy, M. (1973). The Generalized Feynman Integral, International Journal of Theoretical Physics, 8, No. 1, pp. 31-37.

Pardy, M. (2016). Energy Shift of H-Atom Electrons Due to GibbonsHawking Thermal Bath, Journal of High Energy Physics, Gravitation and Cosmology, 2016, 2, 472-477.

Popov, V. S., Kuznetzov, V. P. and Perelomov, A. M. (1968). Quasiclassical Approximation for Nonstationary Problems, JETP, 53, 331.

Welton, T. (1948). Some Observable Effects of the Quantum-Mechanical Fluctuations of the Electromagnetic Field, Physical Review, 74, 1157.

Yamamoto, H. and Kudo, K. (1971). Quantum Field Theory of Complex Mass. II, Progress of Theoretical Physics, 46, No. 4, 1278. 